\section{Leveraging Meteorite Outgassing Experiments to Constrain the Initial Atmospheres of Terrestrial Exoplanets}

\author{
MAGGIE THOMPSON ${ }^{1}$, MYRIAM TELUS ${ }^{2}$, LAURA \\ SCHAEFER $^{3}$, JONATHAN J FORTNEY ${ }^{1}$, TOYANATH \\ JOSHI $^{1}$ AND DAVID LEDERMAN ${ }^{1}$
}

${ }^{1}$ University of California, Santa Cruz

${ }^{2} \mathrm{UCSC}$

${ }^{3}$ Stanford University

Presenting Author: maapthom@ucsc.edu

At present, there is no first-principles understanding of how to connect a planet's bulk composition to its initial atmospheric properties. Since terrestrial exoplanets likely form their atmospheres through outgassing, a novel step towards building such a theory is to assay meteorites, the left-over building blocks of planets, by heating them to measure their outgassed volatiles. Our Solar System presents a wide variety of meteorite types, including carbonaceous chondrites which are believed to be representative of the bulk material in the solar nebula during planet formation. In addition, carbonaceous chondrites contain the highest proportions of volatiles relative to other remnant materials from terrestrial planet formation that can be directly studied in the laboratory. Although planet formation alters planetesimals through thermal and differentiation processes, carbonaceous chondrite-like material was likely an important source of volatiles for the Solar System's terrestrial planets, making these meteorites well-suited for studying early terrestrial exoplanet atmospheres.

To inform the initial chemical composition of terrestrial planet atmospheres, we present the results of our outgassing experiments in which we heated carbonaceous $(\mathrm{CM}, \mathrm{CO}, \mathrm{CV})$ chondrite samples to $1200{ }^{\circ} \mathrm{C}$ and measured the abundances of released volatiles (e.g., $\mathrm{H}_{2} \mathrm{O}, \mathrm{CO}, \mathrm{CO}_{2}, \mathrm{H}_{2}, \mathrm{H}_{2} \mathrm{~S}$ ) as a function of temperature and time. Our experimental set-up consists of a residual gas analyzer, a type of mass spectrometer particularly sensitive to trace amounts of gas, connected to a furnace to heat samples at specified rates. We also perform complementary bulk element analysis on the samples before and after the heating experiments using inductively coupled plasma mass spectrometry to monitor outgassing of heavier elements (e.g., Na, $\mathrm{Mg}, \mathrm{P}, \mathrm{S}, \mathrm{K}, \mathrm{Ca}, \mathrm{Cr}, \mathrm{Mn}, \mathrm{Fe}, \mathrm{Co}, \mathrm{Ni}, \mathrm{Zn}$ ). We compare these experimental results to thermochemical equilibrium models of outgassing from the same types of chondrites and determine how these experiments will improve the atmospheric models. This work presents an experimental framework utilizing chondritic meteorites that takes an important step forward in connecting terrestrial planet interiors and early atmospheres. Ultimately, our results provide a set of experimentally-determined initial conditions for outgassed atmospheric compositions and enable better assumptions to be made in terrestrial exoplanets' initial atmospheres.

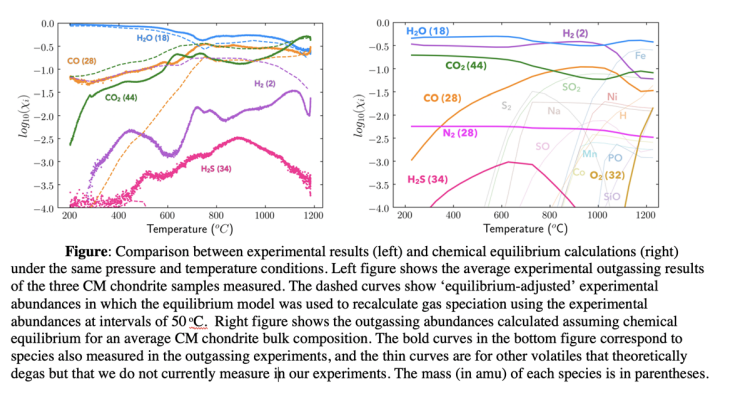

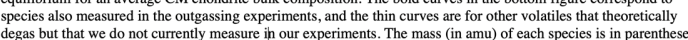

